\title{
End-user need based creation of a medical device : an experience of co-design to struggle pathological scars
}

\author{
Thomas Lihoreau ${ }^{1}$, Brice Chatelain, M.D. ${ }^{2}$, Gwenaël Rolin, PhD. ${ }^{1,3}$, Chrystelle Vidal ${ }^{1}$, Nadia \\ Butterlin, PhD. ${ }^{4}$, Emmanuelle Jacquet, PhD. ${ }^{5}$, Aflah Elouneg 5 , Jérôme Chambert, PhD. ${ }^{5}$, Xavier \\ Bertrand, PharmD, $\mathrm{PhD}^{6}$, Christophe Meyer, M.D., PhD. ${ }^{2,7}$ and Aurélien Louvrier, M.D. ${ }^{2,3}$ \\ ${ }^{1}$ CHU Besançon, INSERM CIC 1431, Centre d'Investigation Clinique, Besançon, France \\ ${ }^{2}$ CHU Besançon, Department of maxillo-facial surgery, Besançon, France \\ ${ }^{3}$ Univ. Bourgogne Franche-Comté, INSERM, EFS BFC, UMR1098, Besançon, France \\ ${ }^{4}$ Univ. Bourgogne Franche-Comté, Institut supérieur d'ingénieurs de Franche-Comté ISIFC, Besançon, France \\ ${ }^{5}$ Univ. Bourgogne Franche-Comté, FEMTO-ST Department of Applied Mechanics, CNRS/UFC/ENSMM/UTBM, Besançon, \\ France \\ ${ }^{6}$ CHU Besançon, Hygiène hospitalière, UMR 6249 Chrono-environnement, Université de Bourgogne-Franche-Comté, \\ Besançon, France \\ ${ }^{7}$ Univ. Bourgogne Franche-Comté, Nanomedicine Lab EA 4662, Besancon, France \\ tlihoreau@chu-besancon.fr
}

Keywords : $\quad$ user-need, keloid scars, medical device, innovation cycle, clinical investigation

\begin{abstract}
Scar is a common visible mark of human tissue healing. Sometimes pathological phenomena lead to abnormal hypertrophic or keloid scars, with evolutions varying depending on different conditions: origin of the tissue barrier disruption, concerned body area, or ethnic origin. Based on these statements, care procedures have been developed to avoid aesthetical or functional impairments: drugs injection, surgery, cryotherapy or mechanical compression. The story will relate the matching of a multi-disciplinary team that focused on covering an unmet need for ear lobe keloid treatment, providing patients an optimal and holistic care. The benefits researched lied in improving the understanding of the disorder, avoiding the recidivism of the scars, diminishing the frequency and duration of care, and in end improving patients' quality of life. The paper will not only narrate the building of a health innovation, on technological, clinical, user points of view, but will also try to detail the evaluations planned at the different stages of development, as well as the challenges, conditions and prerequisites allowing to produce concrete solution.
\end{abstract}

\section{INTRODUCTION - THE MEDICAL PROBLEM}

The keloid scar is defined as a pathology of tissue healing resulting from a proliferation of fibrous tissues that extend beyond the limits of the initial wound (Butler et al., 2008). This pathology, described as "pseudo-cancerous", does not put the patient's vital prognosis into threat, but could constitute a severe aesthetic disturbance in addition to inducing serious functional problems, pain and itching, seriously impacting the quality of life of the patients, especially for scars on visible areas of the skin. Available epidemiological data indicate an incidence that can be very high (16\%) in subjects with ethnic skin (Bayat et al., 2003).

The management of this pathology by the surgeons is difficult and seems randomly addressed. Indeed, different treatments are proposed, ranging from the injection of corticosteroids, to cryotherapy and the administration of anticancer molecules (Ud-Din et al., 2013). At present, no treatment, or combination of treatments, have been described as effective. The main classical management remaining intra keloid resection, it too often leads to a more serious recurrence of keloid in 45 to $100 \%$ of cases (Andrews et al., 2016).

The lack of standard and effective treatment is mainly due to the poor understanding of the cellular and 
tissue mechanisms involved in the appearance and evolution of keloids. For many years homemade compression techniques have been described in the literature to prevent recurrence of keloids after surgery. Especially the handiworks were dedicated to the ear, area of frequent occurrence of this type of pathology, often developed after piercing (Brent et al., 1978, Vachiramon et al., 2004, Chang et al., 2005, Yigit et al. Coll., 2009; Park and Chang, 2013; Tanaydin et al., 2016). The effectiveness of these means of compression relies on the reduction of the post-surgical relapse of the earlobe keloid, observed to a range of 10 to $30 \%$ (Vachiramon et al., 2004, Park and Chang, 2013, Tanaydin et al. 2016).

Even if these works have been presented, there is no current consensus or shared and "gold standard" practice for the treatment of the ear, particularly its compression lobe. One of the causes being the lack of solid clinical trials on the subject (Louis and Gracia, 2010). We proposed then a work assembling from the beginning different experts around the development of a quite unpretentious medical device, which materialize in fact the center of complex considerations.

\section{THE ADVENTURE OF EMERGENCE OF THE IDEA}

In 2014, a surgeon from our university hospital, contacted the clinical investigation research center for a need related to his clinical practice, in fact the medical problem announced in part 1 of this abstract. His difficulty concerned then the reccurrences of keloid scars on an important proportion of his patients, which he yet treated consciensioulsy with intralesional resection plus corticosteroids triamcinolone acetonide injection.

Meeting the research engineers, he explained his needs in a system to add to the current care, relatively to the litterature arguments in favor of a compression of these specific tissues on one point, and to the existing proposed solutions on another point.

At the beginning the deal seemed to be fairly simple: the possibility of adjustment of the pressure by the patient himself (within a limit of the maximum number of magnets imposed by the clinician) would favorize the observance of the device and its comfort. For the few existing studies on the subject, correlation between keloid recurrence of the ear and discomfort in wearing a device has been proved to be correlated (Tanaydin et al, 2016), which may be related to poor adherence to the application of pressure procedure.

Following works in collaborations with other clinicians (to confort the shared property of the expressed need), engineering and business local schools (bibliographic, research \& development, clinical, and market analysis successive training periods), as well as with engineering research center, permitted to formalize a state of the art, and the first drafts of the value analysis and specifications of the innovation, in terms of ergonomy, adaptability, cost, aesthetic...

The collaborations led then to the design of a product as well as evaluations all along the progression.

\section{TECHNOLOGICAL DEVELOPMENTS: THE "SCAR WARS" PROJECT}

Based on brainstorming and on the kind of "Santa letter-writing" desires from the clinicians, but also from the specific anatomical area, and from the technical constraints, the prototypes were first computer-aided designed (Figure 1 left) to format, modelize the idea and project the skateholders into a first view of the possible object.

From that, discussions led - beginning of 2015 - to adaptations before an agreement on the general shape and on primary dimensioning options.

The next step consisted in a 3D printing thanks to stereolithography: few samples of different sizes where produces, manipulated and confronted to the ear lobes of healthy volunteers (from the team...Figure 1 right).

It permitted to define then the size, but also to determine the fact that our idea would need to be constitued of a clip on which magnets could be easily inserted. We had our proof of concept definition prototype.

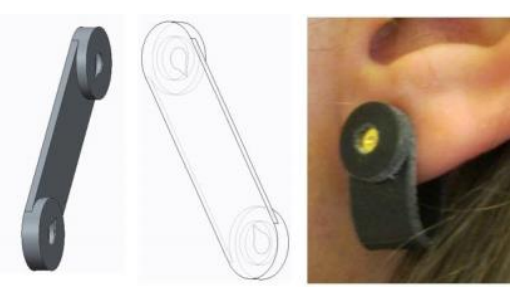

Figure 1: digital and first physical version of the clip 
At the same time, bench lab tests on magnets figured out their sizing - and in fact the possible applied strengths.

A support associated with a dynamometer system measured the forces in work with different magnets and depending of the distance between.

According to the results, for a coherent lobe thickness plus a pressure to be applied (from the litterature) of 25 to $35 \mathrm{mmHg}$, we defined that we would need 2 to 8 neodymium-iron-bore magnets with nickel coating magnets (1mm thickness, diameter $12 \mathrm{~mm}$ ) to be placed on ear lobe.

We needed then to securize the product before thinking of a first use in human. The contact with an industry allowed to produce a mold from which the first clips made with flexible medical grade silicone were manufactured on February 2016 (Figure 2).

In terms of idea protection, an anteriority mark tool was used in December 2016.

It was then the time to think about testing it on targetted concerned patients with keloid scars.

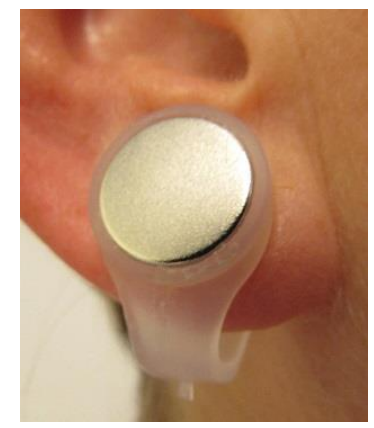

Figure 2: The Scar Wars clip

\section{THE REAL LIFE TEST}

\subsection{Requirements}

In order to provide a product that could be tested during a clinical trial, the responsible manufacturer need to follow regulatory requirements, centralized by the european Medical Device Regulation MDR 2017/745 (repealing Council Directive 93/42/EEC).

Our ambition was to test a product which was not yet CE marked. As a reminder, the CE mark is obtain by a procedure in which notified bodies examine the conformity of the product. On this particular situation (without yet industrial part identified as a owner), our hospital assumed the responsibility as a regulatory manufacturer, for the clinical trial. Actually to obtain the authority agreement to perform the clinical study, we had to provide a file quite similar to the one that would be presented for CE mark obtention.

Thus the team formalize a technical file, including conception plans, laboratory tests, risk analysis, essential requirements answers (list of all applicable standards and the way we addressed it), user manual, labelling and packaging.

This technical file (or "investigator brochure" on a clinical trial language) aim to present the product that will be tested and the security measures taken by the manufacturer to ensure its safety use.

The medical device under study was then defined as related to a Class I according to the requirements of the EC Directive.

On another side, the clinical trial running (or "design") needed to be described in study documents, the master ones being the study protocol, the informed consent and case report forms. A specific budget had also been searched and obtained to finance the clinical project (hospital internal research call).

\subsection{Design of the clinical trial}

The building of the study protocol was an important phase of our project, and its writing needed to mobilize all the partners. It helped to define the objectives, the criteria of evaluations, the targetted population (characteristics and number), the progress in terms of duration... all this taking into account the data already availables (in the litterature and thanks to our previous advances), as well as the previous realized tests and obtained results.

The main objective was defined as the evaluation of the effectiveness and safety of the compressive device; the main endpoint being then the reccurence (yes/no) of the pathology. The study concerned 27 male and female patients (more than 18 years old) presenting keloid lobe ear scars that needed to be treated by reconstructive surgery; it excluded patients with known allergy to nickel (even if the magnets are not in direct contact, silicone clip making the interface).

After usual management of the keloid scar of the ear (reconstructive surgery and injection of corticosteroids - triamcinolone acetonide), the concerned patients had to wear the compressive device and to adjust the compression with the magnets provided. By consulting the literature, which proposes that the patient wears his compression device 8 to 24 hours a day (Louis and Garcia, 2010), 
it was decided to recommend to the patient to apply a compression allowing him to wear the device at least 12 hours a day, daily and throughout the duration of study, ie one year. The compression must be sufficient, without being painful. The clinician will rely on these data, individually for each patient, to dictate the maximum number of magnets to be used based on the measured thickness of the patient's ear. The clinician may reduce the frequency of use of the device, or even stop it according to the evaluation of the quality of healing during visits.

The patients were planned to be seen at 3, 6 and 12 months after intervention, in the traditionnal course of visits during the usual care (no modification due to the trial).

The secondary objectives of the Scar Wars trial focused on a multimodal and interdisciplinary assessment of scar tissues by (Chambert et al., 2019): - evaluation of patient acceptance and satisfaction, evaluation by the surgeon (specific scars evaluation scales, Draaijers et al., 2004, Deslauriers et al., 2009), - biometric characterization of the area of interest,

- non-invasive imaging assessment of tissue evolution,

- analysis of the bacterial flora present at the level of the keloid scar,

- the creation of a keloid cell bank, basis of a biological ancillar study allowing our biologists to focus on pathological healing process and antifibrotic drug evaluation.

\subsection{Official agreements}

The "pilot study evaluating the effectiveness and safety of a compressive device intended to prevent recurrence of keloid scars after surgical resection" file was submitted on February 2017 onto French authorities, with a final positive agreement by ethical committee and national agency for health products ANSM Agence nationale de sécurité du médicament et des produits de santé - obtained in August 2017.

The trial was recorded on official web platform ClinicalTrials.gov, and the first patient was included in October 2017.

To date, 10 patients have been included, without presenting a reccurence.

\section{NEXT STEPS}

The enrolment of the last patients and results of the study will feed the CE mark file, which is then already initiated.

Apart for the CE mark class I obtention, the next important stage will be to build the business model and development associated to an official regulatory manufacturer that will handle the responsibilities and assure the distribution.

Concerning the material, the perspectives could lie on the development of different sizes of the clip and magnets, in order to fit as much as possible to different morphologies, or even other area on ear or even face. 3D printing technologies offer also prefigure tailor-made medical devices. Functionalization with specific drugs or molecules could be the future of such innovations.

In projection, next evaluations could focus for sure on safety aspects once devices will be on the market and largely diffused (material vigilance), and on aggregation and reinforcement of clinical evidences of the innovation. Medico-economics studies will aim to test and possibly prove advantages of the invention relatively to the current costs for patients, hospital, society.

\section{DISCUSSION}

Based on our experience of ideation from a clinical uncovered need, formalization of an innovation, development and testing, we would like to share interesting points that guided us and seems conditions of success for bringing innovation in care and in medical devices field, which guid by definition to complex projects.

\subsection{Guiding principles}

Team effort was a key in our pathway to a concrete solution: clinic, clinical research, technology, regulations, ethics, usability, market / business strategy, intellectual protection, project management... are skills hardly or not often grouped in the context of an hospital, or of medical devices field which is oftenly represented by small medium enterprise.

Contacts need to be actively researched outside, and realized with experts motivated to answer the questions and develop the specific project with 
anticipation and relevancy. Else the innovation risks to encounter the "death valley" located between research and real life.

Related to that, the time and money are important to anticipate. As we speak about little team, we can imagine the consequences of timelines like the ones we presented here, onto the survival of the start-up if not planned with a strong and realistic vision.

\subsection{Some tools?}

Developing innovation in health is a field on which theory and models exist. Well known scales such as Technology Readiness Level (TRL, Scar Wars being today at a TRL 6-7), declined in Market RL, Financial RL... can provide accurate marks for emergence, development, maturity.

We can also refer to more dedicated ones to health such as CREPS cycle (Concept, Research, Evaluation, Product, Care, Moreau-Gaudry A et al. 2010), Innovation RL, health tech innovation cycles (Center for Integration of Medicine and Innovative Technology - CIMIT, Boston) that take into consideration the dimensions of technology, regulatories, market, clinics...

Projects have also been provided on the subject, let's cite for example the European Itech "Roadmap for Research and Innovation in Health Technology" (FP7-HEALTH-2013-INNOVATION-1, CSA-SA https://cordis.europa.eu/project/id/602667/fr), that describe 5 phases from need to industrialization, leading to 5 outcomes from proof of concept (POC) to reimbursment and commercialization.

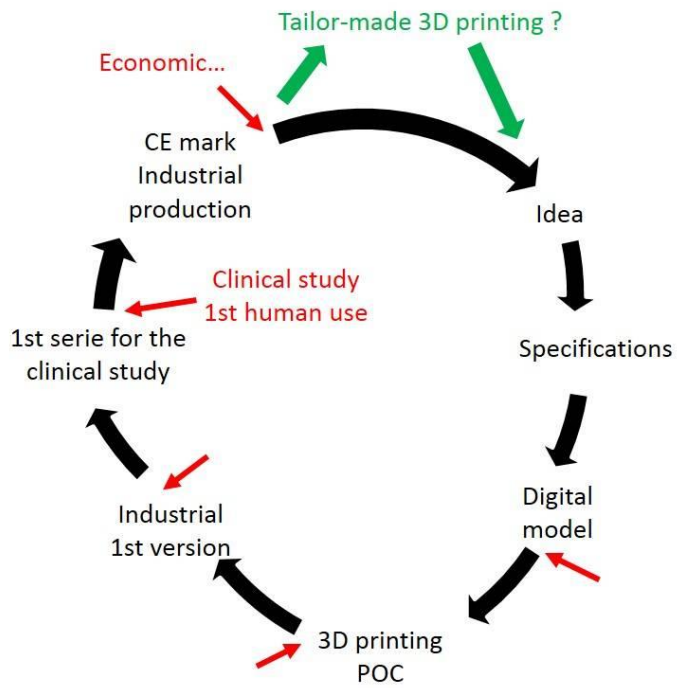

Figure 3: Scar Wars cycle of development
The idea is to try using the scales/tools to define the project roadmap from the beginning, to anticipate the stages, duration, money needed, and to evaluate its progress regularly.

We proposed on Figure 3 the cycle of development of our innovation, with 7 steps from idea to industrialization, evaluations indicated in red, and perspectives in green.

\section{CONCLUSION}

The aim of the SCAR WARS project was to evaluate the effectiveness and safety of an innovation in the treatment of specific pathology on a targeted body location.

The device is proposed in addition to the usual care of patients seen at surgery departments, and will provide, at the level of the lesion, a controlled physical compression, reported in the literature as being a determining factor for the reduction of the volume of the scars and the rate of recurrence after reconstructive surgery.

The conclusions of the work carried out during the project will make it possible to lay solid foundations for the valorization of the device. Above all, the original and multimodal approach of evaluation could help identify new areas of improvement in the pathology management, and provide to the community new scientific data for a better understanding of these scars, and possible successes or failures of proposed treatments.

This first study will quickly provide the patient with an inexpensive device, with targeted properties of aesthetic, comfort and adjustability by the patient himself (within the limit of the maximum number of magnets imposed by the clinician). Those characteristics are hoped to be source of better compliance and therefore efficiency. The expected decrease in recidivism rates could result in a reduction in public health costs for resumption of resection, which could be evaluated with specific methodologies late. Adaptation closer to the morphology of the patient, or to other areas of the body by $3 \mathrm{D}$ printing can then constitute a potential opening of this project.

In terms of a more global approach and for an ambitious project, we tried to enhance the key important guidelines; general schedule of the pathway of innovation could be resumed by: 
-writing of the project and the general objective: state of the art, context (points to improve), positioning, with clinicians and experts in the field;

-describing the solution (s) to be developed, broken down into several lots (technical, regulatory, tests (pre- and clinical, pre- and post-market...), business marketing, etc.), and players to be brought up at each stage.

-formalizing a consortium accordingly: do we have all the internal or external actors identified for the different stages ?

-establishing the budget (and means of search and obtention) accordingly.

One of the first deliverable of any project could be this master roadmap document, adapted and improved all along the life of the project.

\section{REFERENCES}

Andrews JP, Marttala J, Macarak E, Rosenbloom J, Uitto J (2016) Keloids: The paradigm of skin fibrosis -

Pathomechanisms and treatment. Matrix Biol J Int Soc Matrix Biol. 51:37-46.

Bayat A, McGrouther DA, Ferguson MWJ (2003) Skin scarring. BMJ 326:88-92.

Brent B (1978) The role of pressure therapy in management of earlobe keloids: preliminary report of a controlled study. Ann Plast Surg.1:579.

Butler PD, Longaker MT, Yang GP (2008) Current Progress in Keloid Research and Treatment. J Am Coll Surg 206:731-741.

Chambert J, Lihoreau T, Joly S, Chatelain B, Sandoz P, Humbert P, Jacquet E, Rolin G (2019). Multimodal investigation of a keloid scar by combining mechanical tests in vivo with diverse imaging techniques. Journal of the Mechanical Behavior of Biomedical Materials, Volume 99, Pages 206-215.

Chang CH, Song JY, Park JH, Seo SW (2005) The efficacy of magnetic disks for the treatment of earlobe hypertrophic scar. Ann Plast Surg. 54:566-9.

Deslauriers V., Rouleau D.-M., et al. Translation of the Patient Scar Assessment Scale (PSAS) to French with cross-cultural adaptation, reliability evaluation and validation. Can. J. Surg. 2009 52: 259-263.

Draaijers LL, RHF Tempelman, Botman AMY, et al. The Patient and Observer Scar Assessment Scale: a reliable and feasible tool for scar evaluation. Plast Reconstr Surg 2004; 113: 1960-5; discussion 1966-7.

Louis DD, Garcia C (2010) Pressure earring as an adjunct to surgical removal of earlobe keloids. Dermatol Surg. $36: 726$.

Moreau-Gaudry A, Pazart L (2010). Development of an innovation in health: the cycle CREPS Concept, Research, Evaluation, Product, Care. IRBM 31 (1) pp 12-21.

Park TH, Chang CH (2013) Early postoperative magnet application combined with hydrocolloid dressing for the treatment of earlobekeloids. Aesthetic Plast Surg. 37:439-44.

Tanaydin V, Beugels J, Piatkowski A, Colla C, van den Kerckhove E, Hugenholtz GC, van der Hulst RR (2016) Efficacy of custom-made pressure clips for ear keloid treatment after surgical excision. J Plast Reconstr Aesthet Surg.69:115-21.

Ud-Din S, Bayat A (2013) Strategic management of keloid disease in ethnic skin: a structured approach supported by the emerging literature. Br J Dermatol. 169 Suppl 3:71-81.

Vachiramon A, Bamber MA (2004) A U-loop pressure clip for earlobekeloid. J Prosthet Dent.92:389e91.

Yigit B, Yazar M, Alyanak A, Guven E (2009) A custommade silicon mold for pressure therapy to ear keloids.Aesthetic Plast Surg. 33:849-51. 